\title{
Isomorphism Classes and Zeta-functions of Some Nilpotent Groups II
}

\author{
Fumitake HYODO \\ Waseda University \\ (Communicated by N. Suwa)
}

\begin{abstract}
Torsion-free finitely generated nilpotent groups are called $\mathcal{T}$-groups. This article gives a class of $\mathcal{T}$-groups in which zeta functions of groups determine the isomorphism classes.
\end{abstract}

\section{Introduction}

Zeta functions of groups were introduced by F. J. Grunewald, D. Segal, and G. C. Smith [2]: Given a finitely generated group $G$ and a positive integer $n, a_{n}(G)$ denotes the number of subgroups of index $n$. The zeta function of $G$ is defined as the Dirichlet series associated with the sequence $\left\{a_{n}(G)\right\}_{n \geq 1}$, and this function is denoted by $\zeta_{G}$ i.e.,

$$
\zeta_{G}(s):=\sum_{n=1}^{\infty} a_{n}(G) n^{-s}=\sum_{H:[G: H]<\infty}[G: H]^{-s} .
$$

For each prime $p, \zeta_{G, p}(s)$ is also defined as

$$
\zeta_{G, p}(s):=\sum_{k=0}^{\infty} a_{p^{k}}(G) p^{-k s} .
$$

Torsion-free finitely generated nilpotent groups are called $\mathcal{T}$-groups. It is known that zeta functions of $\mathcal{T}$-groups have various properties. F. J. Grunewald, D. Segal, and G. C. Smith [2] proved that if $G$ is a $\mathcal{T}$-group, the zeta function of $G$ has a non-empty domain of convergence and can be expressed as an infinite product:

$$
\zeta_{G}(s)=\prod_{p} \zeta_{G, p}(s)
$$

and for each prime number $p, \zeta_{G, p}(s)$ has a rationality. Moreover, C. Voll [5] showed that for almost all $p, \zeta_{G, p}(s)$ has a functional equation.

Received June 23, 2014; revised December 23, 2014

2010 Mathematics Subject Classification: 11S40 (Primary), 20D15 (Secondary)

Key words and phrases: Zeta functions, nilpotent groups, subgroup growth 
We are interested in finding a class of $\mathcal{T}$-groups in which zeta functions determine the isomorphism classes. It is known that for the class of all $\mathcal{T}$-groups, the isomorphism classes are not determined by zeta functions (cf. [1], Proposition B and [4], Lemma 1.2). Hence we consider a subclass of the above class.

Let $M_{n}\left(\mathbb{Z}^{m}\right)$ denote the set of all $n \times n$ matrices with entries from $\mathbb{Z}^{m}$. For $n, m \in \mathbb{Z}_{\geq 0}$ and $A \in M_{n}\left(\mathbb{Z}^{m}\right)$, a $\mathcal{T}$-group $\left(\mathbb{Z}^{n}, \mathbb{Z}^{m} ; A\right)$ was defined in our previous article [3], Section 3 . We define this group again.

1. As a set we have $\left(\mathbb{Z}^{n}, \mathbb{Z}^{m} ; A\right)=\mathbb{Z}^{n} \times \mathbb{Z}^{m}$.

2. The composition of $(\mathbf{a}, \mathbf{b}),\left(\mathbf{a}^{\prime}, \mathbf{b}^{\prime}\right) \in \mathbb{Z}^{n} \times \mathbb{Z}^{m}$ is defined by

$$
(\mathbf{a}, \mathbf{b})\left(\mathbf{a}^{\prime}, \mathbf{b}^{\prime}\right):=\left(\mathbf{a}+\mathbf{a}^{\prime}, \mathbf{b}+\mathbf{b}^{\prime}+{ }^{t} \mathbf{a} A \mathbf{a}^{\prime}\right) .
$$

For $n \in \mathbb{Z}_{\geq 0}$, we define $\mathfrak{T}_{n}$ to be the class $\left\{\left(\mathbb{Z}^{n}, \mathbb{Z}^{m} ; A\right) \mid m \in \mathbb{Z}_{\geq 0}, A \in M_{n}\left(\mathbb{Z}^{m}\right)\right\}$. It was shown that zeta functions determine the isomorphism classes of $\mathfrak{T}_{n}$ for the case $n=2$ by calculating explicit forms of the zeta functions and that if $n \geq 4$, the result does not hold (cf. [3], Theorem 2 and Remark 3). As far as the author knows, it seems that there are few results on our problem except the above two results. The present article proves the following theorem:

MAIN Theorem. Let $G, G^{\prime} \in \mathfrak{T}_{3}$. Then $\zeta_{G}(s)=\zeta_{G^{\prime}}(s)$ if and only if $G$ and $G^{\prime}$ are isomorphic.

Our main theorem is proved by comparing $a_{l}(G)$ with $a_{l}\left(G^{\prime}\right)$ for some $l$ instead of evaluating their explicit forms (Recall that two sequence $\left\{a_{n}\right\}_{n \geq 1}$ and $\left\{b_{n}\right\}_{n \geq 1}$ are equal if and only if their Dirichlet series are the same.). It is worth pointing out that our result settles the problem whether zeta functions of groups determine the isomorphism classes of the class $\mathfrak{T}_{n}$ for each $n$.

We shall use the notation

- $Z(G):=$ the center of $G$ for an arbitrary group $G$,

- $[G, G]:=$ the commutator subgroup of $G$ for an arbitrary group $G$,

- $h(G):=$ the Hirsch length of $G$ for a $\mathcal{T}$-group $G$,

- $r(G):=$ the $\mathbb{Z}$-ranks of $G$ for a finitely generated abelian group $G$.

(Recall that the Hirsch length of a $\mathcal{T}$-group is defined by the sum of the $\mathbb{Z}$-ranks of its central factors.) Let $n$ be a nonnegative integer, and define $\mathfrak{T}_{n}^{\prime}$ to be the class of $\mathcal{T}$-groups $G$ such that

- the nilpotent class of $G$ is 2 ,

- $r(G / Z(G)) \leq n$,

- $r([G, G]) \leq h(G)-n$.

In fact $\mathfrak{T}_{n}$ is equal to $\mathfrak{T}_{n}^{\prime}$ up to isomorphism. We will prove this in the last section. It is easy to see that this fact deduces:

COROLLARY. Zeta functions of groups determine the isomorphism classes of $\mathfrak{T}_{3}^{\prime}$. 
The outline of this article is as follows. Section 2 classifies isomorphism classes of $\mathfrak{T}_{3}$, and then we reduce to considering a class $\mathfrak{G}$ of $\mathcal{T}$-groups. Section 3 introduces some basic notions and facts to count subgroups of objects of the class $\mathfrak{G}$. In Section 4 , we show that for two groups in the class $\mathfrak{G}$ whose zeta function are the same, their Hirsch lengths are equal. In Section 5, we prove that if two groups in the class $\mathfrak{G}$ have the same Hirsch length and zeta function, then they are isomorphic.

\section{Classification of isomorphism classes}

Let us classify isomorphism classes of $\mathfrak{T}_{n}$. We fix non-negative integers $n, m$. By [3], $\S 3$, we regard $M_{n}\left(\mathbb{Z}^{m}\right)$ as a left- $G L_{m}(\mathbb{Z})$-module and a left and right $G L_{n}(\mathbb{Z})$-module.

We define another right- $G L_{n}(\mathbb{Z})$-module structure on $M_{n}\left(\mathbb{Z}^{m}\right)$ as follows: for $X \in$ $G L_{n}(\mathbb{Z})$ and $A \in M_{n}\left(\mathbb{Z}^{m}\right)$, we put

$$
A \star X:={ }^{t} X A X
$$

Let us define $\operatorname{Alt}_{n}\left(\mathbb{Z}^{m}\right)$ and $\operatorname{Sym}_{n}\left(\mathbb{Z}^{m}\right)$ to be $\left\{A \in M_{n}\left(\mathbb{Z}^{m}\right) \mid{ }^{t} A+A=O\right\}$ and $\{A \in$ $M_{n}\left(\mathbb{Z}^{m}\right) \mid{ }^{t} A-A=O$ \}, respectively. We regard $\operatorname{Alt}_{n}\left(\mathbb{Z}^{m}\right)$ and $\operatorname{Sym}_{n}\left(\mathbb{Z}^{m}\right)$ as $G L_{m}(\mathbb{Z})$ and $G L_{n}(\mathbb{Z})$-submodules of $M_{n}\left(\mathbb{Z}^{m}\right)$. Note that for $A, B \in M_{n}\left(\mathbb{Z}^{m}\right)$ if $G L_{m}(\mathbb{Z}) A=G L_{m}(\mathbb{Z}) B$ or $A \star G L_{n}(\mathbb{Z})=B \star G L_{n}(\mathbb{Z})$ or $A \equiv B \bmod \left(\operatorname{Sym}_{n}\left(\mathbb{Z}^{m}\right)\right)$, then the two groups $\left(\mathbb{Z}^{n}, \mathbb{Z}^{m} ; A\right)$ and $\left(\mathbb{Z}^{n}, \mathbb{Z}^{m} ; B\right)$ are isomorphic (cf. [3], Proposition1 and 2). It is easy to see $M_{n}\left(\mathbb{Z}^{m}\right) / \operatorname{Sym}_{n}\left(\mathbb{Z}^{m}\right)$ is isomorphic to $\operatorname{Alt}_{n}\left(\mathbb{Z}^{m}\right)$ by the map

$$
\left(A \bmod \left(\operatorname{Sym}_{n}\left(\mathbb{Z}^{m}\right)\right) \mapsto A-{ }^{t} A\right)
$$

as left- $G L_{m}(\mathbb{Z})$-modules and right- $G L_{n}(\mathbb{Z})$-modules. Thus by considering the double cosets $G L_{m}(\mathbb{Z}) \backslash \operatorname{Alt}_{n}\left(\mathbb{Z}^{m}\right) / G L_{n}(\mathbb{Z})$, we classify isomorphism classes of groups $\mathfrak{T}_{3}$.

Let us confine ourselves to the case $n=3$. We denote by $M(m, 3 ; \mathbb{Z})$ the set of all $m \times 3$ matrices with entries from $\mathbb{Z} . M(m, 3 ; \mathbb{Z})$ is regarded as a left- $G L_{m}(\mathbb{Z})$-module and a right- $G L_{3}(\mathbb{Z})$-module naturally. It is clear that the map $\tau: \operatorname{Alt}_{3}\left(\mathbb{Z}^{m}\right) \rightarrow M(m, 3 ; \mathbb{Z})$ being

$$
\left(\begin{array}{ccc}
\mathbf{0} & \mathbf{x} & \mathbf{y} \\
-\mathbf{x} & \mathbf{0} & \mathbf{z} \\
-\mathbf{y} & -\mathbf{z} & \mathbf{0}
\end{array}\right) \mapsto\left(\begin{array}{lll}
\mathbf{z} & \mathbf{y} & \mathbf{x}
\end{array}\right)
$$

is isomorphism of left- $G L_{m}(\mathbb{Z})$-modules. For $X \in G L_{3}(\mathbb{Q})$, we denote by $\tilde{X}$ the adjugate matrix of $X$. Let us define a homomorphism $\rho: G L_{3}(\mathbb{Q}) \rightarrow G L_{3}(\mathbb{Q})$ by $(X \mapsto$ $\left.\operatorname{diag}[1,-1,1]^{t}(\tilde{X}) \operatorname{diag}[1,-1,1]\right)$. We remark that $\left.\rho\right|_{G L_{3}(\mathbb{Z})}$ is a surjective homomorphism from $G L_{3}(\mathbb{Z})$ to $S L_{3}(\mathbb{Z})$. By a direct computation, one can check that the maps $\rho$ and $\tau$ are compatible, i.e., for $A \in \operatorname{Alt}_{3}\left(\mathbb{Z}^{m}\right)$ and $X \in G L_{3}(\mathbb{Z})$

$$
\tau(A \star X)=\tau(A) \rho(X) .
$$

Hence we are reduce to giving a complete system of representatives of

$$
G L_{m}(\mathbb{Z}) \backslash M(m, 3 ; \mathbb{Z}) / S L_{3}(\mathbb{Z}),
$$


which is settled by the fundamental theorem of elementary divisors.

We denote by $G(\mathbf{x}, \mathbf{y}, \mathbf{z} ; m)$ the group $\left(\mathbb{Z}^{3}, \mathbb{Z}^{m} ;\left(\begin{array}{ccc}\mathbf{0} & \mathbf{x} & \mathbf{y} \\ \mathbf{0} & \mathbf{0} & \mathbf{z} \\ \mathbf{0} & \mathbf{0} & \mathbf{0}\end{array}\right)\right)$. For non-negative integers $\alpha, \beta, \gamma$, we put

- $G(\alpha, \beta, \gamma ; 0):=G(0,0,0 ; 0):=\mathbb{Z}^{3}$.

- $G(\alpha, \beta, \gamma ; 1):=G(\alpha, 0,0 ; 1):=G\left(\left(\begin{array}{lll}\alpha & 0 & 0\end{array}\right) ; 1\right)$.

- $G(\alpha, \beta, \gamma ; 2):=G(\alpha, \beta, 0 ; 2):=G\left(\left(\begin{array}{ccc}\alpha & 0 & 0 \\ 0 & \beta & 0\end{array}\right) ; 2\right)$.

- $G(\alpha, \beta, \gamma ; 3):=G\left(\left(\begin{array}{ccc}\alpha & 0 & 0 \\ 0 & \beta & 0 \\ 0 & 0 & \gamma\end{array}\right) ; 3\right)$.

- $G(\alpha, \beta, \gamma ; m):=G(\alpha, \beta, \gamma ; 3) \times \mathbb{Z}^{m-3} \quad(m \geq 3)$.

Let us denote by $\mathfrak{G}_{m}$ the class of groups of all $G(\alpha, \beta, \gamma ; m)$ such that

- $\alpha \mathbb{Z} \supset \beta \mathbb{Z} \supset \gamma \mathbb{Z} \quad(m \geq 3)$.

- $\alpha \mathbb{Z} \supset \beta \mathbb{Z}, \gamma=0 \quad(m=2)$.

- $\beta=\gamma=0 \quad(m=1)$.

- $\alpha=\beta=\gamma=0 \quad(m=0)$.

We also define $\mathfrak{G}$ to be the union of the classes $\mathfrak{G}_{m}$ for all non-negative integers $m$. By the above discussion, $\mathfrak{G}$ is a system of representatives of isomorphism classes of $\mathfrak{T}_{3}$.

Proposition 1. Let $A \in M_{3}\left(\mathbb{Z}^{m}\right)$ and $G:=\left(\mathbb{Z}^{3}, \mathbb{Z}^{m} ; A\right)$. Then there exists $G^{\prime} \in \mathfrak{G}_{m}$ such that $G$ is isomorphic to $G^{\prime}$.

In the next three sections, we show that $\mathfrak{G}$ forms a complete system of representatives.

\section{Basic notions and facts for counting subgroups}

In this section, we introduce some basic notions and facts to count subgroups of objects of the class $\mathfrak{G}$. For a group $G$, we write $H \leq G$ (resp. $H \leq{ }_{f} G$ ) if $H$ is a subgroup (resp. a subgroup of finite index) of $G$. For the rest of this section, we fix a prime number $p$ and $G:=G(\alpha, \beta, \gamma ; m) \in \mathfrak{G}_{m}$. One can identify $G$ with the group

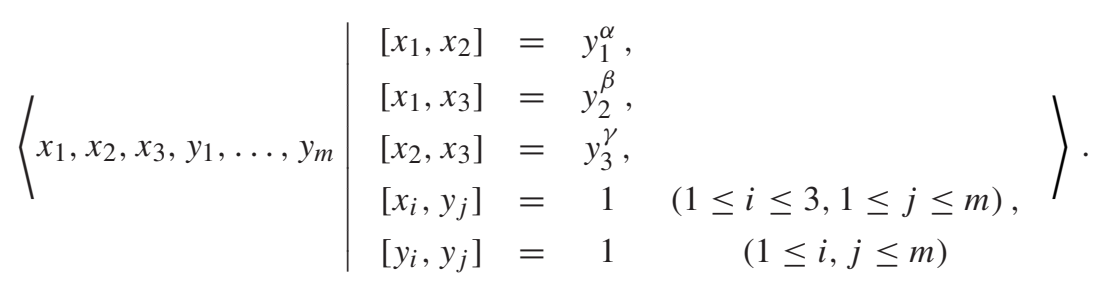


Hence we have a canonical exact sequence:

$$
1 \rightarrow \mathbb{Z}^{m} \stackrel{\iota}{\rightarrow} G \stackrel{\pi}{\rightarrow} \mathbb{Z}^{3} \rightarrow 1
$$

As in [3], §5, for $\mathfrak{h} \leq_{f} \mathbb{Z}^{3}$ and $M \leq_{f} \mathbb{Z}^{m}$, we define $\mathfrak{a}_{G}(\mathfrak{h}, M)$ to be the cardinality of the set $\left\{H \leq G \mid \pi(H)=\mathfrak{h}, \iota^{-1}(H)=M\right\}$. We denote it briefly by $\mathfrak{a}(\mathfrak{h}, M)$. Note that if a subgroup $H$ of $G$ satisfies $\pi(H)=\mathfrak{h}$ and $\iota^{-1}(H)=M$, then $[G: H]=\left[\mathbb{Z}^{3}: \mathfrak{h}\right]\left[\mathbb{Z}^{m}: M\right]$. Let $n \in \mathbb{Z}_{\geq 0}$ and let $\mathfrak{h} \leq \mathbb{Z}^{3}$ satisfy that $\left[\mathbb{Z}^{3}: \mathfrak{h}\right]$ divides $p^{n}$. We put

$$
a_{p^{n}}(G, \mathfrak{h}):=\sum_{M} \mathfrak{a}(\mathfrak{h}, M)
$$

where the sum is taken over all $M$ satisfying $\left[\mathbb{Z}^{3}: \mathfrak{h}\right]\left[\mathbb{Z}^{m}: M\right]=p^{n}$. Then we see

$$
a_{p^{n}}(G)=\sum_{\mathfrak{h}} a_{p^{n}}(G, \mathfrak{h}),
$$

where the sum is taken over all $\mathfrak{h}$ such that $\left[\mathbb{Z}^{3}: \mathfrak{h}\right]$ divides $p^{n}$. By [3], Proposition 3, we have:

$$
a_{p^{n}}(G, \mathfrak{h})=\sum_{M: \mathfrak{a}(\mathfrak{h}, M) \neq 0}\left[\mathbb{Z}^{m}: M\right]^{3},
$$

where the sum is taken over all $M$ satisfying $\left[\mathbb{Z}^{3}: \mathfrak{h}\right]\left[\mathbb{Z}^{m}: M\right]=p^{n}$. By the basis $\left\{\pi\left(x_{1}\right), \pi\left(x_{2}\right), \pi\left(x_{3}\right)\right\}$ of $\mathbb{Z}^{3}$, we can identify the subgroups of finite index in $\mathbb{Z}^{3}$ with $M_{3}(\mathbb{Z}) \cap G L_{3}(\mathbb{Q}) / G L_{3}(\mathbb{Z})$. For $A \in M_{3}(\mathbb{Z}) \cap G L_{3}(\mathbb{Q})$ and a subgroup $\mathfrak{h}$ of $\mathbb{Z}^{3}$, if $\mathfrak{h}$ is generated by the entries of $\left(\pi\left(x_{1}\right) \quad \pi\left(x_{2}\right) \quad \pi\left(x_{3}\right)\right) A$, we say that $\mathfrak{h}$ corresponds to $A$, or that $A$ corresponds to $\mathfrak{h}$. We fix $\mathfrak{h} \leq_{f} \mathbb{Z}^{3}$. Let $A \in M_{3}(\mathbb{Z}) \cap G L_{3}(\mathbb{Q})$ be a matrix corresponding to $\mathfrak{h}$. One can assume that $A$ is a lower triangular matrix. Let us put

$$
A:=\left(\begin{array}{lll}
a & 0 & 0 \\
b & d & 0 \\
c & e & f
\end{array}\right),
$$

and

$$
\left(\begin{array}{lll}
h_{1} & h_{2} & h_{3}
\end{array}\right):=\left(\begin{array}{lll}
x_{1} & x_{2} & x_{3}
\end{array}\right) A
$$

i.e.,

$$
\begin{aligned}
& h_{1}:=x_{1}^{a} x_{2}^{b} x_{3}^{c}, \\
& h_{2}:=x_{2}^{d} x_{3}^{e}, \\
& h_{3}:=x_{3}^{f} .
\end{aligned}
$$


Then $\pi\left(h_{1}\right), \pi\left(h_{2}\right), \pi\left(h_{3}\right)$ is a basis of $\mathfrak{h}$. By [3], Proposition 3, we see that for $M \leq \mathbb{Z}^{m}$, $\mathfrak{a}(\mathfrak{h}, M) \neq 0$ if and only if

$$
\left[h_{1}, h_{2}\right],\left[h_{1}, h_{3}\right],\left[h_{2}, h_{3}\right] \in M .
$$

Let us put $p^{k}:=p^{n} /\left[\mathbb{Z}^{3}: \mathfrak{h}\right]$. Then we have

$$
a_{p^{n}}(G, \mathfrak{h})=p^{3 k} a_{p^{k}}\left(\mathbb{Z}^{m} / N(\mathfrak{h})\right),
$$

where $N(\mathfrak{h}):=\left\langle\left[h_{1}, h_{2}\right],\left[h_{1}, h_{3}\right],\left[h_{2}, h_{3}\right]\right\rangle$. By a direct computation, we have:

$$
\left(\left[h_{2}, h_{3}\right] \quad\left[h_{1}, h_{3}\right] \quad\left[h_{1}, h_{2}\right]\right)=\left(\left[x_{2}, x_{3}\right] \quad\left[x_{1}, x_{3}\right] \quad\left[x_{1}, x_{2}\right]\right) \rho(A),
$$

i.e.,

$$
\left(\left[h_{2}, h_{3}\right] \quad\left[h_{1}, h_{3}\right] \quad\left[h_{1}, h_{2}\right]\right)=\left(\begin{array}{lll}
y_{3}^{\gamma} & y_{2}^{\beta} & y_{1}^{\alpha}
\end{array}\right) \rho(A),
$$

where $\rho$ is defined in the previous section. It is easy to see that

$$
\rho(A)=\left(\begin{array}{ccc}
d f & b f & b e-c d \\
0 & a f & a e \\
0 & 0 & a d
\end{array}\right) .
$$

Note that the above formula is well-defined although $m<3$. For example, if $m=2$, then $y_{3}$ is not defined, but $\gamma=0$. Hence $y_{3}^{\gamma}$ is the identity. Thus we don't have to consider $y_{3}$ in the above formula. Put

$$
\left(\begin{array}{lll}
z_{1} & z_{2} & z_{3}
\end{array}\right):=\left(\begin{array}{lll}
y_{3}^{p^{v_{p}(\gamma)}} & y_{2}^{p^{v_{p}(\beta)}} & y_{1}^{p^{v_{p}(\alpha)}}
\end{array}\right) \rho(A),
$$

where $v_{p}(*)$ means the $p$-adic valuation. Since $\left[\mathbb{Z}^{m}: M\right]$ is $p$-th power, $M$ contains $p^{k} \mathbb{Z}^{m}$ for some $k$. Thus $\mathfrak{a}(\mathfrak{h}, M) \neq 0$ if and only if

$$
z_{1}, z_{2}, z_{3} \in M
$$

Therefore we have the following formulas:

LEMMA 1. Let us put $G_{p}:=G\left(p^{v_{p}(\alpha)}, p^{v_{p}(\beta)}, p^{v_{p}(\gamma)} ; m\right)$. Then

$$
a_{p^{n}}(G)=a_{p^{n}}\left(G_{p}\right) \quad(n \geq 0),
$$

where $v_{p}(*)$ means the $p$-adic valuation.

Lemma 2. Let $n \in \mathbb{Z}_{\geq 0}$, and $\mathfrak{h}$ be a subgroup of $\mathbb{Z}^{3}$ whose index divides $p^{n}$, and let

$$
A:=\left(\begin{array}{lll}
a & 0 & 0 \\
b & d & 0 \\
c & e & f
\end{array}\right)
$$


be a matrix corresponding to $\mathfrak{h}$. Put $p^{k}:=p^{n} /\left[\mathbb{Z}^{3}: \mathfrak{h}\right]$ and

$$
\rho_{G}(A):= \begin{cases}\operatorname{diag}[\gamma, \beta, \alpha] \rho(A) & (m \geq 3), \\
\operatorname{diag}[\beta, \alpha]\left(\begin{array}{cc}
a f & a e \\
0 & a d
\end{array}\right) & (m=2), \\
\alpha a d & (m=1) .\end{cases}
$$

Then

$$
a_{p^{n}}(G, \mathfrak{h})=p^{3 k} \begin{cases}a_{p^{k}}\left(\mathbb{Z}^{m-3} \times \mathbb{Z}^{3} / \rho_{G}(A) \mathbb{Z}^{3}\right) & (m \geq 3), \\ a_{p^{k}}\left(\mathbb{Z}^{2} / \rho_{G}(A) \mathbb{Z}^{2}\right) & (m=2), \\ a_{p^{k}}\left(\mathbb{Z} / \rho_{G}(A) \mathbb{Z}\right) & (m=1) .\end{cases}
$$

\section{The case $m \neq m^{\prime}$}

In this section we shall prove:

Proposition 2. Let $G \in \mathfrak{G}_{m}$ and $G^{\prime} \in \mathfrak{G}_{m^{\prime}}$. If $m \neq m^{\prime}$, then for some prime $p$,

$$
\zeta_{G, p}(s) \neq \zeta_{G^{\prime}, p}(s) .
$$

By the above proposition, we have:

Corollary 1. Let $G \in \mathfrak{G}_{m}$ and $G^{\prime} \in \mathfrak{G}_{m^{\prime}}$. If $\zeta_{G}(s)=\zeta_{G^{\prime}}(s)$, then $m=m^{\prime}$.

To prove Proposition 2, it is sufficient to prove:

Proposition 3. Let $G$ and $G^{\prime}$ be as in Proposition 2. Then for some prime number p,

$$
\left(a_{p}(G), a_{p^{2}}(G)\right) \neq\left(a_{p}\left(G^{\prime}\right), a_{p^{2}}\left(G^{\prime}\right)\right) .
$$

We fix a prime number $p$ and $G:=G(\alpha, \beta, \gamma ; m) \in \mathfrak{G}_{m}$. Now we evaluate $a_{p}(G)$ and $a_{p^{2}}(G)$.

PROPOSITION 4. The following formulas hold:

$$
a_{p}(G)= \begin{cases}a_{p}\left(\mathbb{Z}^{m+3}\right) & (p \mid \alpha), \\ a_{p}\left(\mathbb{Z}^{m+2}\right) & (p \nmid \chi \alpha, p \mid \beta), \\ a_{p}\left(\mathbb{Z}^{m+1}\right) & (p \nmid \alpha, p \nmid \beta, p \mid \gamma), \\ a_{p}\left(\mathbb{Z}^{m}\right) & (p \nmid \chi \alpha, p \nmid \beta, p \nmid \gamma) .\end{cases}
$$

ProOF. We see that

$$
a_{p}(G)=a_{p}\left(G, \mathbb{Z}^{3}\right)+\sum_{\mathfrak{h}:\left[\mathbb{Z}^{3}: \mathfrak{h}\right]=p} a_{p}(G, \mathfrak{h}) .
$$


Assume $\left[\mathbb{Z}^{3}: \mathfrak{h}\right]=p$. For a subgroup $M$ of $\mathbb{Z}^{m}$, if $\left[\mathbb{Z}^{3}: \mathfrak{h}\right]\left[\mathbb{Z}^{m}: M\right]=p$, then $\mathbb{Z}^{m}=M$. Hence $\mathfrak{a}(\mathfrak{h}, M) \neq 0$. Thus

$$
\sum_{\mathfrak{h}:\left[\mathbb{Z}^{3}: \mathfrak{h}\right]=p} a_{p}(G, \mathfrak{h})=a_{p}\left(\mathbb{Z}^{3}\right) .
$$

Assume $\mathfrak{h}=\mathbb{Z}^{3}$. We evaluate $a_{p}\left(G, \mathbb{Z}^{3}\right)$. For a subgroup $M$ of $\mathbb{Z}^{m}$ satisfying $\left[\mathbb{Z}^{3}: \mathfrak{h}\right]\left[\mathbb{Z}^{m}\right.$ : $M]=p, \mathfrak{a}(\mathfrak{h}, M) \neq 0$ if and only if

$$
y_{1}^{\alpha}, y_{2}^{\beta}, y_{3}^{\gamma} \in M,
$$

Since $\left[\mathbb{Z}^{m}: M\right]=p$,

$$
p \mathbb{Z}^{m} \subset M .
$$

Put $N:=\left\langle y_{1}^{\alpha}, y_{2}^{\beta}, y_{3}^{\gamma}\right\rangle+p \mathbb{Z}^{m}$. Then by the definition of $a_{p}\left(G, \mathbb{Z}^{3}\right)$,

$$
a_{p}\left(G, \mathbb{Z}^{3}\right)=p^{3} a_{p}\left(\mathbb{Z}^{m} / N\right) .
$$

$\mathbb{Z}^{m} / N$ is isomorphic to

$$
\begin{cases}\mathbb{F}_{p}^{m} & (p \mid \alpha) \\ \mathbb{F}_{p}^{m-1} & (p \nmid \alpha, p \mid \beta) \\ \mathbb{F}_{p}^{m-2} & (p \nmid \chi \alpha, p \nmid \beta, p \mid \gamma) \\ \mathbb{F}_{p}^{m-3} & (p \nmid \alpha, p \nmid \beta, p \quad \backslash \gamma) .\end{cases}
$$

Since $a_{p}\left(\mathbb{F}_{p}^{k}\right)=a_{p}\left(\mathbb{Z}^{k}\right)$ for $k \in \mathbb{Z}_{\geq 0}$,

$$
a_{p}(G)=a_{p}\left(\mathbb{Z}^{3}\right)+p^{3} \begin{cases}a_{p}\left(\mathbb{Z}^{m}\right) & (p \mid \alpha), \\ a_{p}\left(\mathbb{Z}^{m-1}\right) & (p \nmid \chi \alpha, p \mid \beta), \\ a_{p}\left(\mathbb{Z}^{m-2}\right) & (p \nmid \chi \alpha, p \nmid \beta, p \mid \gamma), \\ a_{p}\left(\mathbb{Z}^{m-3}\right) & (p \nmid \chi \alpha, p \nmid \beta, p \nmid \gamma) .\end{cases}
$$

By [3], Corollary 3 , for $l, k \in \mathbb{Z}_{\geq 0}$

$$
a_{p}\left(\mathbb{Z}^{l+k}\right)=a_{p}\left(\mathbb{Z}^{l}\right)+p^{l} a_{p}\left(\mathbb{Z}^{k}\right),
$$

which completes the proof.

Proposition 5. The following formulas hold:

$$
a_{p^{2}}(G)= \begin{cases}a_{p^{2}}\left(\mathbb{Z}^{m}\right)+p^{m}(1+p)\left(1+p+p^{2}\right) & (G=G(1,1,1 ; m)), \\ a_{p^{2}}\left(\mathbb{Z}^{m+1}\right)+p^{m+1}(1+p)^{2} & (G=G(1,1,0 ; m)), \\ a_{p^{2}}\left(\mathbb{Z}^{m+2}\right)+p^{m+2}(1+p) & (G=G(1,0,0 ; m)), \\ a_{p^{2}}\left(\mathbb{Z}^{m+3}\right) & (G=G(0,0,0 ; m)) .\end{cases}
$$


Proof. Note that

$$
a_{p^{2}}(G)=a_{p^{2}}\left(G, \mathbb{Z}^{3}\right)+\sum_{\mathfrak{h}:\left[\mathbb{Z}^{3}: \mathfrak{h}\right]=p} a_{p^{2}}(G, \mathfrak{h})+\sum_{\mathfrak{h}:\left[\mathbb{Z}^{3}: \mathfrak{h}\right]=p^{2}} a_{p^{2}}(G, \mathfrak{h}) .
$$

An argument similar to the one in the proof of Proposition 4 shows that

1. $a_{p^{2}}(G, \mathfrak{h})=1$ for all $\mathfrak{h}$ such that $\left[\mathbb{Z}^{3}: \mathfrak{h}\right]=p^{2}$.

2. $a_{p^{2}}\left(G, \mathbb{Z}^{3}\right)=p^{6} a_{p^{2}}\left(\mathbb{Z}^{m} / N\right)$, where $N:=\left\langle y_{1}^{\alpha}, y_{2}^{\beta}, y_{3}^{\gamma}\right\rangle$.

\section{Hence}

1.

$$
\sum_{\mathfrak{h}:\left[\mathbb{Z}^{3}: \mathfrak{h}\right]=p^{2}} a_{p^{2}}(G, \mathfrak{h})=a_{p^{2}}\left(\mathbb{Z}^{3}\right)
$$

2.

$$
a_{p^{2}}\left(\mathbb{Z}^{m} / N\right)= \begin{cases}a_{p^{2}}\left(\mathbb{Z}^{m-3}\right) & (G=G(1,1,1 ; m)), \\ a_{p^{2}}\left(\mathbb{Z}^{m-2}\right) & (G=G(1,1,0 ; m)), \\ a_{p^{2}}\left(\mathbb{Z}^{m-1}\right) & (G=G(1,0,0 ; m)), \\ a_{p^{2}}\left(\mathbb{Z}^{m}\right) & (G=G(0,0,0 ; m)) .\end{cases}
$$

Let us evaluate $a_{p^{2}}(G, \mathfrak{h})$ for $\mathfrak{h}$ such that $\left[\mathbb{Z}^{3}: \mathfrak{h}\right]=p$. and let $\Delta_{p}$ denote the set of all elements of $M_{3}(\mathbb{Z}) \cap G L_{3}(\mathbb{Q})$ whose determinants are $\pm p$. It is easy to see that the following set forms a complete system of representatives of $\Delta_{p} / G L_{3}(\mathbb{Z})$ :

$$
\left\{\left(\begin{array}{ccc}
p & 0 & 0 \\
0 & 1 & 0 \\
0 & 0 & 1
\end{array}\right)\right\} \cup\left\{\left(\begin{array}{lll}
1 & 0 & 0 \\
b & p & 0 \\
0 & 0 & 1
\end{array}\right)\right\}_{0 \leq b<p} \cup\left\{\left(\begin{array}{lll}
1 & 0 & 0 \\
0 & 1 & 0 \\
c & e & p
\end{array}\right)\right\}_{0 \leq c, e<p} .
$$

We put

$$
X(0):=\left(\begin{array}{ccc}
p & 0 & 0 \\
0 & 1 & 0 \\
0 & 0 & 1
\end{array}\right), \quad Y(b):=\left(\begin{array}{ccc}
1 & 0 & 0 \\
b & p & 0 \\
0 & 0 & 1
\end{array}\right), \quad Z(c, e):=\left(\begin{array}{ccc}
1 & 0 & 0 \\
0 & 1 & 0 \\
c & e & p
\end{array}\right) .
$$

Then $\rho(X(0)), \rho(Y(b))$ and $\rho(Z(c, e))$ are

$$
\left(\begin{array}{lll}
1 & 0 & 0 \\
0 & p & 0 \\
0 & 0 & p
\end{array}\right), \quad\left(\begin{array}{ccc}
p & b & 0 \\
0 & 1 & 0 \\
0 & 0 & p
\end{array}\right), \quad\left(\begin{array}{ccc}
p & 0 & -c \\
0 & p & e \\
0 & 0 & 1
\end{array}\right),
$$

respectively. Put $\Gamma:=G L_{3}(\mathbb{Z})$. Since $\alpha|\beta| \gamma$, we have

$$
\Gamma \rho_{G}(X(0)) \Gamma=\Gamma \operatorname{diag}[\gamma, p \beta, p \alpha] \Gamma,
$$




$$
\begin{gathered}
\Gamma \rho_{G}(Y(b)) \Gamma=\Gamma \operatorname{diag}[p \gamma, \beta, p \alpha] \Gamma, \\
\Gamma \rho_{G}(Z(c, e)) \Gamma=\Gamma \operatorname{diag}[p \gamma, p \beta, \alpha] \Gamma .
\end{gathered}
$$

Let us assume $m \geq 3$. If $\mathfrak{h}$ corresponds to $X(0)$, then by Lemma 2

$$
a_{p^{2}}(G, \mathfrak{h})=p^{3} \begin{cases}a_{p}\left(\mathbb{Z}^{m-1}\right) & (G=G(1,1,1 ; m)), \\ a_{p}\left(\mathbb{Z}^{m}\right) & (G=G(1,1,0 ; m)), \\ a_{p}\left(\mathbb{Z}^{m}\right) & (G=G(1,0,0 ; m)), \\ a_{p}\left(\mathbb{Z}^{m}\right) & (G=G(0,0,0 ; m)) .\end{cases}
$$

If $\mathfrak{h}$ corresponds to $Y(b)$, then

$$
a_{p^{2}}(G, \mathfrak{h})=p^{3} \begin{cases}a_{p}\left(\mathbb{Z}^{m-1}\right) & (G=G(1,1,1 ; m)) \\ a_{p}\left(\mathbb{Z}^{m-1}\right) & (G=G(1,1,0 ; m)) \\ a_{p}\left(\mathbb{Z}^{m}\right) & (G=G(1,0,0 ; m)) \\ a_{p}\left(\mathbb{Z}^{m}\right) & (G=G(0,0,0 ; m))\end{cases}
$$

If $\mathfrak{h}$ corresponds to $Z(c, e)$, then

$$
a_{p^{2}}(G, \mathfrak{h})=p^{3} \begin{cases}a_{p}\left(\mathbb{Z}^{m-1}\right) & (G=G(1,1,1 ; m)) \\ a_{p}\left(\mathbb{Z}^{m-1}\right) & (G=G(1,1,0 ; m)) \\ a_{p}\left(\mathbb{Z}^{m-1}\right) & (G=G(1,0,0 ; m)) \\ a_{p}\left(\mathbb{Z}^{m}\right) & (G=G(0,0,0 ; m)) .\end{cases}
$$

If $m \leq 2$, then the same formulas hold. (The details are left to the reader.) Note that $\left|\{Y(b)\}_{0 \leq b<p}\right|=p,\left|\{Z(c, e)\}_{0 \leq c, e<p}\right|=p^{2}$. Therefore $a_{p^{2}}(G)$ is equal to

$$
a_{p^{2}}\left(\mathbb{Z}^{3}\right)+ \begin{cases}p^{3} a_{p}\left(\mathbb{Z}^{m-1}\right)+p^{4} a_{p}\left(\mathbb{Z}^{m-1}\right)+p^{5} a_{p}\left(\mathbb{Z}^{m-1}\right)+p^{6} a_{p^{2}}\left(\mathbb{Z}^{m-3}\right) & (G=G(1,1,1 ; m)), \\ p^{3} a_{p}\left(\mathbb{Z}^{m}\right)+p^{4} a_{p}\left(\mathbb{Z}^{m-1}\right)+p^{5} a_{p}\left(\mathbb{Z}^{m-1}\right)+p^{6} a_{p^{2}}\left(\mathbb{Z}^{m-2}\right) & (G=G(1,1,0 ; m)), \\ p^{3} a_{p}\left(\mathbb{Z}^{m}\right)+p^{4} a_{p}\left(\mathbb{Z}^{m}\right)+p^{5} a_{p}\left(\mathbb{Z}^{m-1}\right)+p^{6} a_{p^{2}}\left(\mathbb{Z}^{m-1}\right) & (G=G(1,0,0 ; m)), \\ p^{3} a_{p}\left(\mathbb{Z}^{m}\right)+p^{4} a_{p}\left(\mathbb{Z}^{m}\right)+p^{5} a_{p}\left(\mathbb{Z}^{m}\right)+p^{6} a_{p^{2}}\left(\mathbb{Z}^{m}\right) & (G=G(0,0,0 ; m)) .\end{cases}
$$

By [3], Corollary 3 and induction on $k$, we see that $a_{p}\left(\mathbb{Z}^{k}\right)=\frac{1-p^{k}}{1-p}$ for $k \geq 0$, especially $a_{p}\left(\mathbb{Z}^{3}\right)=1+p+p^{2}$. By [3], Corollary 3 we have

$$
a_{p^{2}}\left(\mathbb{Z}^{k+3}\right)=a_{p^{2}}\left(\mathbb{Z}^{3}\right)+p^{3} a_{p}\left(\mathbb{Z}^{3}\right) a_{p}\left(\mathbb{Z}^{k}\right)+p^{6} a_{p^{2}}\left(\mathbb{Z}^{k}\right) \quad(k \geq 0) .
$$

Thus if $G=G(1,1,1 ; m)$, then

$$
a_{p^{2}}(G)-a_{p^{2}}\left(\mathbb{Z}^{m}\right)=p^{3} a_{p}\left(\mathbb{Z}^{3}\right)\left(a_{p}\left(\mathbb{Z}^{m-1}\right)-a_{p}\left(\mathbb{Z}^{m-3}\right)\right) .
$$


Since

$$
a_{p}\left(\mathbb{Z}^{m-1}\right)-a_{p}\left(\mathbb{Z}^{m-3}\right)=\frac{p^{m-3}-p^{m-1}}{1-p}
$$

we have

$$
a_{p^{2}}(G)=a_{p^{2}}\left(\mathbb{Z}^{m}\right)+p^{m}(1+p)\left(1+p+p^{2}\right) .
$$

If $G \neq G(1,1,1 ; m)$, it suffices to compute

$$
\begin{cases}a_{p^{2}}(G)-a_{p^{2}}\left(\mathbb{Z}^{m+1}\right) & (G=G(1,1,0 ; m)), \\ a_{p^{2}}(G)-a_{p^{2}}\left(\mathbb{Z}^{m+2}\right) & (G=G(1,0,0 ; m)), \\ a_{p^{2}}(G)-a_{p^{2}}\left(\mathbb{Z}^{m+3}\right) & (G=G(0,0,0 ; m)),\end{cases}
$$

similarly.

Let us put

$$
\mathfrak{X}_{m}:=\{G(1,1,1 ; m), G(1,1,0 ; m), G(1,0,0 ; m), G(0,0,0 ; m)\} .
$$

Corollary 2. For $G \in \mathfrak{X}_{m}$ and $G^{\prime} \in \mathfrak{X}_{m^{\prime}}$, if $m>m^{\prime}$ and $a_{p}(G)=a_{p}\left(G^{\prime}\right)$ then

$$
a_{p^{2}}(G)>a_{p^{2}}\left(G^{\prime}\right) .
$$

Proof. We assume $G=G(1,1,1 ; m)$. By Proposition $4, G^{\prime}=G(1,1,0 ; m-1)$, or $G^{\prime}=G(1,0,0 ; m-2)$ or $G^{\prime}=G(0,0,0 ; m-3)$. By Proposition 5, in all three cases, we see that $a_{p^{2}}(G)>a_{p^{2}}\left(G^{\prime}\right)$. The proof for the case $G \neq G(1,1,1 ; m)$ is similar

Now we show Proposition 3.

Proof. We may assume that $m>m^{\prime}$ and $a_{p}(G)=a_{p}\left(G^{\prime}\right)$ for all $p$.

Let $G:=G(\alpha, \beta, \gamma ; m)$ and $G^{\prime}:=G\left(\alpha^{\prime}, \beta^{\prime}, \gamma^{\prime} ; m^{\prime}\right)$. For some $p$, the $p$-adic valuations of $\alpha, \beta, \gamma, \alpha^{\prime}, \beta^{\prime}$, and $\gamma^{\prime}$ are contained in $\{0, \infty\}$. By Lemma 1 we reduced to the case $G \in \mathfrak{X}_{m}$ and $G^{\prime} \in \mathfrak{X}_{m^{\prime}}$. By Corollary 2, we have $a_{p^{2}}(G)>a_{p^{2}}\left(G^{\prime}\right)$.

\section{The case $m=m^{\prime}$}

In this section, we complete the proof of our main theorem. We fix $m \in \mathbb{Z}_{\geq 0}$. First, we prove the following lemma:

Lemma 3. Let $n \in \mathbb{Z}_{\geq 0}$, and $G, G^{\prime} \in \mathfrak{G}_{m}$. For all $\mathfrak{h} \leq \mathbb{Z}^{3}$ and $M \leq \mathbb{Z}^{m}$ such that $\left[\mathbb{Z}^{3}: \mathfrak{h}\right]\left[\mathbb{Z}^{m}: M\right]=n$, we suppose that

$$
\text { if } \mathfrak{a}_{G}(\mathfrak{h}, M) \neq 0 \text {, then } \mathfrak{a}_{G^{\prime}}(\mathfrak{h}, M) \neq 0 \text {. }
$$

Then

$$
a_{n}(G) \leq a_{n}\left(G^{\prime}\right) .
$$


Moreover, under the above condition, if there exist $\mathfrak{h} \leq \mathbb{Z}^{3}, M \leq \mathbb{Z}^{m}$ such that $\left[\mathbb{Z}^{3}: \mathfrak{h}\right]\left[\mathbb{Z}^{m}\right.$ : $M]=n, \mathfrak{a}_{G}(\mathfrak{h}, M)=0$ and $\mathfrak{a}_{G^{\prime}}(\mathfrak{h}, M) \neq 0$, then

$$
a_{n}(G)<a_{n}\left(G^{\prime}\right) .
$$

Proof. By [3], Proposition 3, for all $\mathfrak{h} \leq \mathbb{Z}^{3}, M \leq \mathbb{Z}^{m}$ such that $\left[\mathbb{Z}^{3}: \mathfrak{h}\right]\left[\mathbb{Z}^{m}: M\right]=$ $n$, we have

$$
\mathfrak{a}_{G}(\mathfrak{h}, M) \leq \mathfrak{a}_{G^{\prime}}(\mathfrak{h}, M),
$$

which implies

$$
a_{n}(G) \leq a_{n}\left(G^{\prime}\right) .
$$

Moreover, if there exist $\mathfrak{h} \leq \mathbb{Z}^{3}, M \leq \mathbb{Z}^{m}$ such that $\left[\mathbb{Z}^{3}: \mathfrak{h}\right]\left[\mathbb{Z}^{m}: M\right]=n, \mathfrak{a}_{G}(\mathfrak{h}, M)=0$ and $\mathfrak{a}_{G^{\prime}}(\mathfrak{h}, M) \neq 0$, then

$$
\mathfrak{a}_{G}(\mathfrak{h}, M)<\mathfrak{a}_{G^{\prime}}(\mathfrak{h}, M),
$$

which implies

$$
a_{n}(G)<a_{n}\left(G^{\prime}\right)
$$

This completes the proof.

Now we shall prove the following proposition:

Proposition 6. Let $G:=G(\alpha, \beta, \gamma ; m)$ and $G^{\prime}:=G\left(\alpha^{\prime}, \beta^{\prime}, \gamma^{\prime} ; m\right)$ be elements of $\mathfrak{G}_{m}$. If $(\alpha, \beta, \gamma) \neq\left(\alpha^{\prime}, \beta^{\prime}, \gamma^{\prime}\right)$, then for some $n$,

$$
a_{n}(G) \neq a_{n}\left(G^{\prime}\right) .
$$

By the above proposition, we have:

COROllary 3. Let $G$ and $G^{\prime}$ as above. If $\zeta_{G}(s)=\zeta_{G^{\prime}}(s)$, then $(\alpha, \beta, \gamma)=$ $\left(\alpha^{\prime}, \beta^{\prime}, \gamma^{\prime}\right)$.

Now we shall prove Proposition 6.

Proof. By Lemma 1, we reduce to the case where $\alpha, \beta, \gamma, \alpha^{\prime}, \beta^{\prime}, \gamma^{\prime}$, and $n$ are $p$-th powers. Let us put

$$
\left(\alpha, \beta, \gamma, \alpha^{\prime}, \beta^{\prime}, \gamma^{\prime}\right):=\left(p^{i}, p^{j}, p^{k}, p^{i^{\prime}}, p^{j^{\prime}}, p^{k^{\prime}}\right),
$$

where $\left(0 \leq i \leq j \leq k \leq \infty, 0 \leq i^{\prime} \leq j^{\prime} \leq k^{\prime} \leq \infty\right)$. Note that by assumption, $\left(p^{i}, p^{j}, p^{k}\right) \neq\left(p^{i^{\prime}}, p^{j^{\prime}}, p^{k^{\prime}}\right)$. We put

$$
l:= \begin{cases}i & \left(i<i^{\prime}\right) \\ j & \left(i=i^{\prime}, j<j^{\prime}\right), \\ k & \left(i=i^{\prime}, j=j^{\prime}, k<k^{\prime}\right) .\end{cases}
$$


It is sufficient to show that $a_{p^{l+1}}(G) \neq a_{p^{l+1}}\left(G^{\prime}\right)$ for the above three cases. Let $\mathfrak{h} \leq \mathbb{Z}^{3}$ and $M \leq \mathbb{Z}^{m}$ satisfy $\left[\mathbb{Z}^{3}: \mathfrak{h}\right]\left[\mathbb{Z}^{m}: M\right]=p^{l+1}$. And let $A$ be a matrix corresponding to $\mathfrak{h}$. We put

$$
\left(\begin{array}{lll}
z_{1} & z_{2} & z_{3}
\end{array}\right):=\left(\begin{array}{lll}
y_{3}^{p^{k}} & y_{2}^{p^{j}} & y_{1}^{p^{i}}
\end{array}\right) \rho(A),
$$

and

$$
\left(\begin{array}{lll}
z_{1}^{\prime} & z_{2}^{\prime} & z_{3}^{\prime}
\end{array}\right):=\left(\begin{array}{lll}
y_{3}^{p^{k^{\prime}}} & y_{2}^{p^{j^{\prime}}} & y_{1}^{p^{i^{\prime}}}
\end{array}\right) \rho(A)
$$

We recall that

$$
\mathfrak{a}_{G}(\mathfrak{h}, M) \neq 0 \text { if and only if } z_{1}, z_{2}, z_{3} \in M,
$$

and

$$
\mathfrak{a}_{G^{\prime}}(\mathfrak{h}, M) \neq 0 \text { if and only if } z_{1}^{\prime}, z_{2}^{\prime}, z_{3}^{\prime} \in M .
$$

1. The case where $\left[\mathbb{Z}^{m}: M\right]$ divides $p^{l}$.

Since $M \supset p^{l} \mathbb{Z}^{m}, z_{1}, z_{2}, z_{3} \in M$ if and only if $z_{1}^{\prime}, z_{2}^{\prime}, z_{3}^{\prime} \in M$.

2. The case $\left[\mathbb{Z}^{m}: M\right]=p^{l+1}$.

Since $\mathbb{Z}^{3}=\mathfrak{h}$,

$$
\mathfrak{a}_{G}(\mathfrak{h}, M) \neq 0 \text { if and only if } y_{3}^{p^{k}}, y_{2}^{p^{j}}, y_{1}^{p^{i}}, \in M
$$

and

$$
\mathfrak{a}_{G^{\prime}}(\mathfrak{h}, M) \neq 0 \text { if and only if } y_{3}^{p^{k^{\prime}}}, y_{2}^{p^{j^{\prime}}}, y_{1}^{p^{i^{\prime}}}, \in M,
$$

Hence if $\mathfrak{a}_{G}(\mathfrak{h}, M) \neq 0$, then $\mathfrak{a}_{G^{\prime}}(\mathfrak{h}, M) \neq 0$. On the other hand, we take

$$
M:= \begin{cases}\left\langle y_{3}, y_{2}, y_{1}^{p^{l+1}}\right\rangle & \left(i<i^{\prime}\right), \\ \left\langle y_{3}, y_{2}^{p^{l+1}}, y_{1}\right\rangle & \left(i=i^{\prime}, j<j^{\prime}\right), \\ \left\langle y_{3}^{p^{l+1}}, y_{2}, y_{1}\right\rangle & \left(i=i^{\prime}, j=j^{\prime}, k<k^{\prime}\right) .\end{cases}
$$

Then we have $\mathfrak{a}_{G}(\mathfrak{h}, M)=0$, and $\mathfrak{a}_{G^{\prime}}(\mathfrak{h}, M) \neq 0$.

By Lemma 3, we have $a_{p^{l+1}}(G)<a_{p^{l+1}}\left(G^{\prime}\right)$, which completes the proof.

By Proposition 1, Corollary 1, and Corollary 3, we have the following result:

Theorem 1. Let $G, G^{\prime} \in \mathfrak{T}_{3}$. Then $\zeta_{G}(s)=\zeta_{G^{\prime}}(s)$ if and only if $G$ and $G^{\prime}$ are isomorphic.

Finally, we prove $\mathfrak{T}_{n}$ is equal to $\mathfrak{T}_{n}^{\prime}$ up to isomorphism for each $n \in \mathbb{Z}_{\geq 0}$. ( $\mathfrak{T}_{n}^{\prime}$ was defined in Section 1 of this article.)

Proposition 7. For a $\mathcal{T}$-group $G$, there exists $G^{\prime} \in \mathfrak{T}_{n}$ such that $G$ is isomorphic to $G^{\prime}$ if and only if there exists $G^{\prime} \in \mathfrak{T}_{n}^{\prime}$ such that $G$ is isomorphic to $G^{\prime}$. 
Proof. We put $G:=\left(\mathbb{Z}^{n}, \mathbb{Z}^{m} ; A\right)$ for some $n, m \in \mathbb{Z}_{\geq 0}, A \in M_{n}\left(\mathbb{Z}^{m}\right)$. By [3], Section $2, G$ is of nilpotent class 2 , and the subgroup $N:=\left\{(\mathbf{0}, \mathbf{b}) \mid \mathbf{b} \in \mathbb{Z}^{m}\right\}$ of $G$ satisfies

$$
[G, G] \subset N \subset Z(G) .
$$

Since $G / N \cong \mathbb{Z}^{n}$ and $N \cong \mathbb{Z}^{m}$, we have

- $h(G)=n+m$,

- $r(G / Z(G)) \leq r(G / N)=n$,

- $r([G, G]) \leq r(N)=m$.

Hence $r([G, G]) \leq h(G)-n$. Thus $G \in \mathfrak{T}_{n}^{\prime}$.

Let us assume $G \in \mathfrak{T}_{n}^{\prime}$, and let $(k, l):=(h(G), r(G / Z(G)))$. Then there is a Malcev basis $\left\{x_{1}, x_{2}, \ldots, x_{k}\right\}$ of $G$. We put $H_{i}:=\left\langle x_{i}, \ldots, x_{k}\right\rangle(1 \leq i \leq k)$. Recall that $\left\{x_{1}, x_{2}, \ldots, x_{k}\right\}$ is called a Malcev basis of $G$ if $\left\{H_{i} \mid(1 \leq i \leq k)\right\}$ is a lower central series of $G$ and $H_{i} / H_{i+1} \cong \mathbb{Z}(1 \leq i \leq k-1)$. Since $G$ is of nilpotent class 2, for all elements $x, y$ of $G$ and for all integers $k,\left[x^{k}, y\right]=[x, y]^{k}$,where $[x, y]$ means the commutator of $x$ and $y$. Hence $G / Z(G)$ is torsion free, namely an abelian $\mathcal{T}$-group. Thus we may assume that $x_{1}, x_{2}, \ldots, x_{l} \notin Z(G)$ and $H_{l+1}=Z(G)$. Since $[G, G] \subset Z(G)$ and $r([G, G]) \leq k-n$, we may assume that $[G, G] \subset H_{n+1}$. Let $\theta$ be the canonical isomorphism $H_{n+1}$ to $\mathbb{Z}^{k-n}$. Since the nilpotent class of $G$ is 2 , the map $f: \mathbb{Z}^{n} \times \mathbb{Z}^{n} \longrightarrow \mathbb{Z}^{k-n}$ being $\left(\alpha_{1}, \ldots, \alpha_{n}, \beta_{1}, \ldots, \beta_{n}\right) \mapsto \theta\left(\left[x_{1}^{\alpha_{1}} \cdots x_{n}^{\alpha_{n}}, x_{1}^{\beta_{1}} \cdots x_{n}^{\beta_{n}}\right]\right)$ is a bilinear form. Let $A$ be the matrix corresponding to the bilinear form $f$, then we construct an isomorphism $G \rightarrow G\left(\mathbb{Z}^{n}, \mathbb{Z}^{k-n} ; A\right)$ by $\left(x_{1}^{\alpha_{1}} \cdots x_{k}^{\alpha_{k}} \mapsto\left(\alpha_{1}, \ldots, \alpha_{k}\right)\right)$, which completes the proof.

Therefore, we have:

COROLlaRY 4. Zeta functions of groups determine the isomorphism classes of $\mathfrak{T}_{3}^{\prime}$.

ACKNOWLEDGMENT. This research is supported by Waseda University Grant for Special Research Projects 2013A-910.

\section{References}

[ 1 ] F. J. GRUnewAld and R. Scharlau, A note on finitely generated torsion-free groups of class 2, J. Algebra 58 (1979), 162-175.

[ 2 ] F. J. Grunewald, D. Segal and G. C. Smith, Subgroups of finite index in nilpotent groups, Invent. Math. 93 (1988), 185-223.

[ 3 ] F. Hyodo, Isomorphism classes and Zeta-functions of some Nilpotent Groups, Tokyo J. Math. 36 (2013), $163-175$.

[ 4 ] P. F. PICKEL, Finitely generated nilpotent groups with isomorphic finite quotients, Trans. Amer. Math. Soc. 160 (1971), 327-341.

[ 5 ] C. Voll, Functional equations for zeta functions of groups and rings, Ann. of Math. 172 (2010), no. 2, 1181-1218. 
Present Address:

DePartment of APPlied Mathematics,

WASEDA UNIVERSITY,

OKUBO 3-4-1, SINJUKU-KU, TOKYO 169-8555, JAPAN.

e-mail: fumitake.hyodo@aoni.waseda.jp 